\title{
Effects of a national genomic preselection on the international genetic evaluations
}

\author{
Clotilde Patry, ${ }^{*} \dagger^{1}$ Hossein Jorjani, $\ddagger$ and Vincent Ducrocq* \\ *UMR 1313 Génétique Animale et Biologie Intégrative, Institut National de la Recherche Agronomique, 78352 Jouy-en-Josas, France \\ †Union nationale des Coopératives d'Elevage et d'Insémination Animale, 149 rue de Bercy, 75595 Paris Cédex 12, France \\ łInterbull Centre, Department of Animal Breeding and Genetics, Swedish University of Agricultural Sciences, Box 7023, S-75007 Uppsala, \\ Sweden
}

\section{ABSTRACT}

Genomic preselection of young bulls is now widely implemented in dairy breeding schemes, especially in the Holstein breed. However, if this step is not accounted for in genetic evaluation models, the national breeding values of bulls retained by a genomic preselection and of their progeny are estimated with bias. It follows that countries participating in international genetic evaluations will provide a selected and possibly biased set of data to the Interbull Centre (Swedish University of Agricultural Sciences, Uppsala, Sweden). The objective of the study was to show evidence of bias at the international level due to a genomic preselection step in national breeding schemes. The consequence of a genomic preselection for the international evaluations (i.e., using selected and biased national estimated breeding values) was simulated using actual national estimated breeding values as a proxy for genomically enhanced breeding values. Data were provided for 3 countries with a large population of Holstein bulls. International breeding values from simulated scenarios were compared with international breeding values using all available data, assumed to be complete and unbiased. Bias was measured among young bulls retained by a genomic preselection and their contemporaries in other countries. The results were analyzed by traits measured within each country and by country of origin of the young bulls. It turned out that sending preselected data, though based on genomic information, created bias in international evaluations, penalizing young bulls from the country sending the incorrect data. It also had an effect on the young bulls from the other countries. Sending biased data further affected the quality of international evaluations. This study underlines the importance of accounting for genomic preselection at the national level first. Moreover, submitting all available data appeared essential to maintain the quality of the

Received September 27, 2011.

Accepted January 31, 2013.

${ }^{1}$ Corresponding author: clotilde.patry@jouy.inra.fr international genetic evaluations after implementation of a genomic preselection step.

Key words: Interbull, international genetic evaluation, genomic preselection, selection bias

\section{INTRODUCTION}

Breeding strategies in dairy cattle are being transformed by the emergence of genetic evaluation tools combining new molecular technologies and advanced statistical analyses. Genomic selection is developing fast and propagating worldwide. In 2009, only a few countries computed genomically enhanced breeding values (GEBV) in the Holstein breed only; in 2011, data from 5 dairy breeds and 13 countries were provided for validation of national genomic evaluations at the Interbull Centre (Swedish University of Agricultural Sciences, Uppsala, Sweden; Loberg et al., 2011).

Since 1994, the Interbull Centre has been in charge of the routine international genetic evaluations that facilitate comparisons between bull breeding values across countries and promote international genetic exchanges. As proposed by Schaeffer (1994), international EBV (I-EBV) are computed from national EBV (N-EBV) using BLUP applied to a mixed linear sire model for multiple traits. The method is commonly known as multiple-trait across country evaluation (MACE). For the same selection objective, each trait evaluated in different countries can have different levels of heritability so that traits are considered to be different but correlated. It follows that international evaluations benefit from an increased amount of performances for bulls having progeny in several countries. Each bull gets a revised breeding value expressed in the unit of trait used in each participating country. Thus, bulls from all countries can be ranked according to the same base and scale in each country.

The recent changes in national evaluation systems, and the new breeding strategies due to genomic advances, have to be considered in international genetic evaluations to keep providing international comparisons of dairy bulls across a large number of countries. The 
first challenge for the Interbull Centre is to develop a new evaluation methodology using genomic information. The second is to maintain the international genetic evaluations as they are currently delivered, without genomic information and only based on pedigree and phenotypes. The international evaluations is still useful for all of the countries participating in international genetic evaluations, for those not computing genomic evaluations (i.e., 15 out of the 28 countries), and for those with genomic evaluations. In fact, international evaluations provide a tool for fair international comparison that is not yet available based on genomic information. Moreover, I-EBV are not only required to include foreign information in classical national evaluations, but also in the genomic evaluation system. To make genomic evaluations more reliable, the reference population might include phenotyped and genotyped animals from foreign countries.

However, the implementation of a genomic preselection (GPS) step for young bulls (YB) at the national level may threaten the correctness of international genetic evaluations. Two reasons for this exist. The first reason is that, in the near future, each country implementing GPS might send to the Interbull Centre biased data for bulls retained by GPS. Patry and Ducrocq (2011 b) showed by simulations that once the YB selected from genomic information have daughters, their BLUP solutions are, on average, underestimated when compared with the simulated true breeding values. The risk is that such bias could be propagated at the international level to related animals and correlated traits. Biased N-EBV may be avoided if all genotyped and nongenotyped animals are included in the BLUP evaluations. Two approaches might be implemented in national evaluations. The first one is based on singlestep evaluations where the relationship matrix is modified to include all animals, genotyped or phenotyped. (Legarra et al., 2009, Misztal et al., 2009, Aguilar et al., 2010, Christensen and Lund, 2010). The second type of approach is based on multi-step evaluations where GEBV are computed first and then included as weighted-deregressed performances (Ducrocq and Liu, 2009, Patry and Ducrocq, 2011a) besides the actual ones in BLUP evaluations. The GEBV can also be considered as a correlated trait (Mäntysaari and Strandén, 2010, Stoop et al., 2011) for all genotyped candidates. All of these approaches better describe the process of GPS and avoid EBV from being biased; however, all BLUP solutions will include genomic information from that moment.

The second reason is that countries might send to the Interbull Centre performances for selected bulls only and BLUP solutions for culled candidates would be missing. Selection based on genomic information was shown to affect the distribution of the Mendelian sampling (MS) term (Patry and Ducrocq, 2011b) and violate the BLUP hypotheses assuming that the MS term averages to zero. It is thus feared that MACE results computed from a selected subpopulation (e.g., selected YB) could also be biased (Henderson, 1975) too.

Because of those 2 reasons, it was justified to wonder the effect of selected N-EBV on international evaluations. The objective of this study was to describe and assess the effect of GPS on international evaluations due to (1) the propagation of bias from the national to the international level and (2) the creation of a bias in MACE solutions while using selected N-EBV. Three types of GPS strategies could be implemented by the participating countries and were considered for this study. Countries implementing GPS might deliver biased and selected N-EBV to the Interbull Centre (strategy 1). Countries might account for GPS in their national evaluation model and prevent N-EBV from being biased. Therefore, countries might send unbiased N-EBV for either only selected YB (strategy 2) or for all YB (strategy 3) to the Interbull Centre. The bias (i.e., the systematic under- or overestimation of I-EBV) was therefore measured under the alternative strategies, which were simulated based on real data. Consequences on rankings for international comparisons were also analyzed. Solutions to implement at the national and international levels and preventing biased I-EBV were put forward.

\section{MATERIALS AND METHODS}

\section{Material}

To participate in the international evaluations, each country provided the Interbull Centre N-EBV information on performance and their effective daughter contribution as weight. The national data sets required to run the August 2010 routine evaluation were used to simulate GPS and to assess its effect on the international level. In the present study, we focused on one production trait (i.e., protein yield in the Holstein breed), restricted to animals measured and evaluated in only 3 large countries, denoted hereafter as "country A," "country B," and "country C." A total of 57,688 bulls were considered out of the about 116,000 bulls with protein yield evaluations from 27 countries. The 3 countries in this study delivered national genetic evaluations from BLUP applied to a single-trait animal model. Note that country A used a random regression test day model, whereas countries $\mathrm{B}$ and $\mathrm{C}$ used a repeatability model.

Genetic parameters are displayed in Table 1. Heritability values were sent by the national evaluation 
Table 1. Characteristics of evaluation models for protein yield: choice of model, heritability, average equivalent daughter contribution (EDC) among young bulls, estimated sire standard deviation on the diagonal, and genetic correlations between countries below the diagonal

\begin{tabular}{llcccc}
\hline \multirow{2}{*}{ Country } & National genetic & & Young bulls & \\
evaluation model & Heritability & EDC & Trait A & Trait B & Trait C \\
\hline A & Single-trait BLUP animal model - Test day & $48 \%$ & 97.28 & 8.600 & \\
B & Single-trait BLUP animal repeatability model & $30 \%$ & 77.41 & $85 \%$ & 9.165 \\
C & Single-trait BLUP animal repeatability model & $30 \%$ & 97.02 & $87 \%$ & $90 \%$ \\
\hline
\end{tabular}

centers. Genetic correlations among countries and sire standard deviations were computed from previous international evaluations.

For each individual, I-EBV and parental average breeding values were estimated by MACE using the international pedigree and N-EBV from the 3 countries. The I-EBV were expressed on each trait scale according to the genetic evaluation for protein yield in each country. Here, traits refer to measurements of the same biological character in different countries. Thus, the Interbull Centre considers one trait per country to perform a multi-trait evaluation. It follows that simulations and analyses were performed for traits A, B, and $\mathrm{C}$, respectively.

\section{General Strategy}

The I-EBV for the control scenario (CTL) were computed from MACE based on the actual set of N-EBV available at the Interbull Centre for the 3 countries. The N-EBV were assumed to be complete and unbiased (i.e., all candidates to progeny test participated in the international evaluations). Next, the I-EBV were computed from MACE after a simulated GPS step among YB (i.e., after altering the set of N-EBV in size and possibly in value). For that purpose, 2 steps were implemented: (1) the preselection of the YB to (2) participate in the international evaluation (i.e., the deregression of N-EBV followed by the MACE run). The MACE solutions were then compared between the control and the simulated scenarios to assess the bias magnitude in I-EBV due to GPS.

Two types of scenarios were simulated in a repeated way to mimic strategies which could be implemented by the different Interbull members:

(1) Scenario SELU for preselected and unbiased NEBV: GPS was accounted for in national evaluation models and both selected and culled YB received adjusted N-EBV. However, performances for the culled ones were not sent to the Interbull Centre so that national data sets of N-EBV were unbiased but incomplete. In that case, the problem is of using selected data-based genomic information in a multi-trait BLUP model.
(2) Scenarios SELB for preselected and biased NEBV: GPS was not accounted for in national evaluation models. The N-EBV for culled bulls were not available and N-EBV among the preselected bulls were biased. In that case, the specific problem is of using biased data in a multi-trait BLUP model.

According to the simulated scenario, the actual data sets provided to Interbull Centre by countries A, B, and $\mathrm{C}$ were modified (1) by deleting N-EBV among the youngest bulls to mimic a preselection step (scenarios SELU and SELB) and (2) by possibly adding a term of error to N-EBV as if the latter were biased due to the GPS not accounted into BLUP (scenario SELB only).

\section{Mimicking a GPS of the YB}

Before starting, the cohort of candidates for GPS was identified according to 2 criteria: bulls were born during the most recent birth years (i.e., between 2003 and 2006) and had no daughter in any foreign country. The YB cohort included 7,118 bulls and 2,234 had N-EBV for A trait, 1,282 had N-EBV for B trait, and 3,602 had N-EBV for C trait.

Decisions of preselection were assumed to be based on genomic information under the form of GEBV. Compared with the classical estimation of breeding values based on performances and pedigree, the genomic evaluations bring early and accurate information on the MS term and allow ranking of bulls within their cohort of sibs. Before bulls have progeny, the MS term can be computed as the difference between GEBV and parental average $(\mathrm{PA})$ :

$$
M S_{i}=G E B V_{i}-P A_{i} .
$$

In our study, PA values were provided by MACE solutions (I-PA), but GEBV were not available at the Interbull Centre, in contrast with N-EBV. The latter do not include any genomic information but were considered to be equivalent to GEBV for the selection of YB, except that even N-EBV based on first batch of daughters had too high of reliabilities compared with early GEBV. To mimic lower reliability in GEBV, a 
random variable proportional to the breeding value reliability for each bull, $i$ (see Equation 2), was added to the actual MS term:

$$
M S_{i}^{*}=\left(N-E B V_{i}-I-P A_{i}\right)+\text { error }_{i},
$$

where

$$
\text { error }_{i} \sim N\left(0, \lambda \times R_{i}^{2} \times \sigma_{a}^{2}\right),
$$

with $R_{i}^{2}=$ the reliability associated with each $N-E B V_{i}$, $\sigma_{a}^{2}=$ the genetic variance of the trait, and $\lambda=0.75$, so that the reliabilities of N-EBV for YB with first crop of daughters were dropped to a level of about $50 \%$, corresponding to a GEBV reliability at birth.

To make the selection, the simulated MS values $\left(M S^{*}\right)$ were sorted within half-sib families of YB in each country. This was defined as MS scenarios.

A random (RD) preselection of YB was also implemented. Instead of sorting half-sibs based on their MS term, selection was performed within half-sib family according to a random value. In that case, the average MS term of the YB participating in the international evaluations was not supposed to deviate from zero and I-EBV were not expected to be biased. The MACE solutions under an $\mathrm{RD}$ preselection were used to control the effect of reducing the quantity of information involved in the international evaluations and to distinguish it from the effect of using preselected data based on genomic information. This was defined as RD scenarios.

In MS and RD scenarios, selection was only implemented in large families (i.e., bull families including more than 10 half-sibs). According to the MS or RD ranking, the top $10 \%$ of YB within each family were retained. The N-EBV of culled bulls were removed from the file of observations used in the international evaluations. This deletion step systematically occurred in both types of simulated scenarios (SELU and SELB) to consider $90 \%$ of the candidates as missing. Any N-EBV were deleted among the smaller families of YB.

\section{Simulating Bias in N-EBV After a GPS Not Accounted for in the National Evaluation Models}

In case GPS was ignored at the national level, NEBV were assumed to be biased downwards. Therefore, bias was introduced at the national level in N-EBV using the results from the study of Patry and Ducrocq (2011 b). In that study, a GPS step was simulated in the cohort of YB with only one crop of daughters. For a trait with $36 \%$ heritability and a $10 \%$ preselection rate, the bias in the $\mathrm{YB}$ cohort was on average -0.227 in genetic SD of the trait, with a variance of $0.016^{2}$.
Hence, in the present study the individual bias $\left(\Delta_{i}\right)$ was drawn from this normal distribution. This random value, multiplied by the SD of each trait $\left(\sigma_{a}\right)$, was then added to each actual national breeding value, $N-E B V_{i}^{A C T}$, and the sum, $N-E B V_{i}^{S I M}$, was considered as the new input for MACE, only in the case of MS SELB scenarios:

$$
N-E B V_{i}^{S I M}=N-E B V_{i}^{A C T}+\sigma_{a} \times \Delta_{i} .
$$

\section{Implementation}

According to the country implementing a GPS strategy ( $\mathrm{A}, \mathrm{B}$, or $\mathrm{C}$ ), the national evaluation model (accounting for GPS into BLUP or not), and the chosen selection criteria (MS or RD), 9 scenarios were simulated (MS SELU-A, -B, or -C; RD SELU-A, -B, or -C; and MS SELB-A, -B, or -C); they were replicated 10 times. In the first step, 10 different lists of YB per scenario (i.e., 90 different lists in total) were defined. The corresponding N-EBV were used as performances in the international evaluations; the N-EBV of the culled ones were deleted. The actual N-EBV were included in scenarios MS and RD SELU, whereas simulated N-EBV $\left(N-E B V_{i}^{S I M}\right.$, mimicking underestimated N-EBV after a GPS) were provided in scenarios MS SELB only. The CTL scenario was not replicated, as no random sampling was involved; all YB participated in the international evaluations based on their actual NEBV. In the second step, a total of 90 MACE runs were performed. The MACE solutions from the simulated scenarios were finally compared with the MACE solutions in the CTL run to highlight a systematic error of estimation (i.e., a bias due to GPS). The differences between scenarios in the implementation of these 2 steps were summarized in Table 2.

\section{Bias Assessments}

After the simulation of the 9 scenarios, all I-EBV were expressed in genetic SD of each trait. Bias was then estimated as the average difference over all YB $(n)$ between individual $(i)$ standardized I-EBV from the CTL scenario $\left(I-E B V_{i}^{C T L}\right)$ and individual standardized I-EBV from one simulated scenario $\left(I-E B V_{i}^{S I M}\right)$. This was finally averaged over all replicates $(r)$, as follows (Equation 5):

$$
\hat{b}=\frac{1}{10 \times n} \sum_{r=1}^{10} \sum_{i=1}^{n} \frac{I-E B V_{i, r}^{C T L}-I-E B V_{i, r}^{S I M}}{\sigma_{a}} .
$$

Estimates of bias were computed for each trait (A, B, or C) and among each cohort of YB (from country A, 
Table 2. Implemented steps for the simulation of the different scenarios

\begin{tabular}{|c|c|c|c|c|}
\hline \multirow[b]{2}{*}{ Step } & \multicolumn{3}{|c|}{ Simulated scenarios } & \multirow{2}{*}{$\begin{array}{l}\text { Actual } \\
\text { (control) } \\
\text { scenario }\end{array}$} \\
\hline & MS SELU ${ }^{1}$ & RD SELU ${ }^{2}$ & MS SELB ${ }^{3}$ & \\
\hline \multicolumn{5}{|l|}{ Preselection of the young bulls (YB) } \\
\hline Definition of the selection criteria & $\begin{array}{l}\text { The Mendelian sampling term within } \\
\text { half-sib family (MS*) }\end{array}$ & $\begin{array}{l}\text { A random value within half-sib } \\
\text { family }\left(\mathrm{RD}^{*}\right)\end{array}$ & $\begin{array}{l}\text { The Mendelian sampling term within } \\
\text { half-sib family }\left(\mathrm{MS}^{*}\right)\end{array}$ & $\varnothing$ \\
\hline Simulation of the selection criteria ${ }^{4}$ & $\begin{array}{l}M S_{i}^{*}=\left(N-E B V_{i}-P A_{i}\right)+\text { error }_{i} \text { with } \\
\text { error }_{i} \sim N\left(0, \lambda \times R_{i}^{2} \times \sigma_{e}^{2}\right)\end{array}$ & $R D_{i}^{*} \sim N(0,1)$ & $\begin{array}{l}M S_{i}^{*}=\left(N-E B V_{i}-P A_{i}\right)+\text { error }_{i} \text { with } \\
\text { error }_{i} \sim N\left(0, \lambda \times R_{i}^{2} \times \sigma_{e}^{2}\right)\end{array}$ & $\varnothing$ \\
\hline Selection of the YB & \multicolumn{3}{|c|}{$\begin{array}{l}\text { (1) Sorting according to the selection criteria; (2) identifying the top } 10 \% \text { of the YB; (3) removing the N-EBV of the } \\
\text { other } 90 \% \text { from the file of performances }\end{array}$} & $\varnothing$ \\
\hline \multirow{2}{*}{$\begin{array}{l}\text { International evaluation run } \\
\text { MACE input (regarding YB) }\end{array}$} & \multirow{2}{*}{ Actual N-EBV: $N-E B V_{i}^{A C T}$} & \multirow[b]{2}{*}{ Actual N-EBV: $N-E B V_{i}^{A C T}$} & & \\
\hline & & & $\begin{array}{l}\text { Simulated N-EBV: } \\
N-E B V_{i}^{S I M}=N-E B V_{i}^{A C T}+\sigma_{\text {trait }} \times \Delta_{i} \\
\text { with } \Delta_{i} \sim N\left(-0.227,0.016^{2}\right)\end{array}$ & $\begin{array}{l}\text { Actual N-EBV: } \\
N-E B V_{i}^{A C T}\end{array}$ \\
\hline MACE output (regarding YB) ${ }^{6}$ & $I-E B V_{i}^{S I M}$ & $I-E B V_{i}^{S I M}$ & $I-E B V_{i}^{S I M}$ & $I-E B V_{i}^{C T L}$ \\
\hline
\end{tabular}

${ }^{1} \mathrm{MS}$ SELU $=$ one country (A, B, or C) sent to Interbull Centre preselected data after genetic preselection (for only $10 \%$ of young bulls).

${ }^{2} \mathrm{RD}$ SELU $=$ one country (A, B, or C) sent to Interbull Centre randomly preselected data (for only $10 \%$ of young bulls).

${ }^{3} \mathrm{MS}$ SELB = one country (A, B, or C) sent to Interbull Centre preselected and biased data after genetic preselection (for only $10 \%$ of young bulls).

${ }^{4} \mathrm{~N}-\mathrm{EBV}=$ national EBV as multiple-trait across country evaluation solutions. $P A=$ parental average; $R_{i}^{2}=$ the reliability associated with each $N$ - $E B V_{i} ; \sigma_{a}^{2}=$ the genetic variance of the trait; and $\lambda=0.75$

${ }^{5} \Delta_{i}=$ individual bias; $\sigma_{\text {trait }}=$ genetic standard deviation of the trait of interest.

$\mathrm{I}-\mathrm{EBV}=$ international EBV as multiple-trait across country evaluation (MACE) solutions. 
$\mathrm{B}$, or $\mathrm{C}$ ). Bias was also measured among the sires of $\mathrm{YB}$ and their half-sibs.

In the simulations, 2 types of variance for the bias estimates existed: one between animals within a population and one between the $10 \mathrm{MACE}$ runs due to the repeated draw of each YB N-EBV. To account for this latter type of variability that the simulations introduced artificially, bias estimates were adjusted for the variance between repetitions $\left(\sigma_{r}^{2}\right)$ beside the size of the cohorts of interest ( $n$; Equation 6$)$ :

$$
\hat{b}_{A D J}=\frac{\hat{b}}{\sqrt{\sigma_{r}^{2} /(10 \times n)}} .
$$

Bias estimates $(\hat{b})$ and their adjusted values $\left(\hat{b}_{A D J}\right)$ provide 2 types of indication. The bias estimates provide information on how large bias is in real conditions relative to the SD of the considered trait. Conversely, $\hat{b}_{A D J}$ is useful to compare the magnitude and direction of bias across scenarios, cohorts, and traits, in spite of differences of population size and genetic parameters between countries.

Bias estimates and their adjusted values were first compared with a zero mean to characterize the circumstances for biased MACE solutions (trait, country of origin, GPS strategy). To explain the magnitude and direction of the bias if it differed from zero, 3 effects were required to be distinguished to explain differences in the estimation of breeding values:

- Effect $1\left(\mathrm{E}_{1}\right)$ : the reduction of the size of the $\mathrm{YB}$ cohort due to a preselection step in comparison with the larger size of the YB cohort after a progeny test;

- Effect $2\left(\mathrm{E}_{2}\right)$ : the MS deviation from the zero mean after a GPS;

- Effect $3\left(\mathrm{E}_{3}\right)$ : the transmission of a systematic error of estimation from the national level to the international one.

In the RD SELU scenarios, only the effect of type 1 might be involved and the estimated bias $\hat{b}^{R D S E L U}$ can be associated to the estimation of $\mathrm{E}_{1}$ :

$$
\hat{b}^{R D S E L U}=\hat{E}_{1} .
$$

On the contrary, the effects of type 1 and 2 might be responsible for bias in the MS SELU scenarios and

$$
\hat{b}^{M S S E L U}=\hat{E}_{1}+\hat{E}_{2} .
$$

The equality of means under both scenarios $\left(\mathrm{H}_{0}: \hat{b}^{M S S E L U}=\hat{b}^{R D S E L U}\right)$ was tested to distinguish $\mathrm{E}_{1}$ from $\mathrm{E}_{2}$; under $\mathrm{H}_{0}$, the effect of type 2 would be null.

In addition to the bias estimate observed in MS SELU scenarios, $\mathrm{E}_{3}$ might also be involved in MS SELB scenarios. As an approximation, it was assumed that the 3 effects were additive so that

$$
\begin{aligned}
& \hat{b}^{M S S E L B}=\hat{E}_{1}+\hat{E}_{2}+\hat{E}_{3}, \\
\Leftrightarrow & \hat{b}^{M S S E L B}=\hat{b}^{M S S E L U}+\hat{E}_{3}, \\
\Leftrightarrow & \hat{E}_{3}=\hat{b}^{M S S E L B}-\hat{b}^{M S S E L U} .
\end{aligned}
$$

This allows better understanding of the origin of bias in MACE solutions and identifies the most harmful issue for international evaluations.

Finally, to assess the potential effect of bias on selection decisions, the changes in the top 100 ranked bulls were identified and broken down into country of origin and age group (YB vs. proven bulls). For each evaluated trait in country $\mathrm{A}, \mathrm{B}$, or $\mathrm{C}$, and for each scenario, the proportion of YB from country A, B, or C among the top 100 ranked bulls was compared with the proportion observed under the CTL situation.

\section{RESULTS}

\section{Mimicking GPS and Consequences on MS Distribution}

Genomic preselection was mimicked among YB by retaining the bulls with the highest MS estimate within large half-sib families in each of the 3 countries. Table 3 describes the average MS estimate among YB and for each trait in 3 cases: before selection (all candidates were considered, CTL scenario), after a random selection (scenario RD SELU), and after a selection based on MS mimicking GPS (scenario MS SELU). When YB were selected at random (RD SELU), only the quantity of information involved in MACE evaluations was affected. In such cases, as expected, the average MS deviation was found to be close to the zero, and not significantly different from the average MS deviation observed in the CTL scenario. When the GPS was mimicked (scenarios MS SELU), the observed MS mean was significantly different from the zero observed in the CTL scenario for all traits. In MS and RD SELU scenarios, MS was drawn based on an increased variance. Therefore, we cannot compare the SD of the MS term in simulated scenarios with the SD in CTL scenario. On the contrary, we can compare the SD of the MS 
Table 3. Mean and standard deviation of the Mendelian sampling (MS) terms among young bulls $(\mathrm{n}=$ size of each cohort) from country A, B, and C for the trait evaluated in their country of origin according to the implemented scheme ${ }^{1}$ in unit of genetic standard deviation

\begin{tabular}{|c|c|c|c|c|}
\hline \multirow[b]{2}{*}{ Cohort } & \multirow{2}{*}{$\begin{array}{l}\text { Reliability of } \\
\text { mimicked GEBV }\end{array}$} & \multicolumn{3}{|c|}{ Average MS term used to select young bulls } \\
\hline & & CTL & RD SELU ${ }^{3}$ & MS SELU ${ }^{4}$ \\
\hline Young bulls from $\mathrm{A}$ & $51 \%$ & $\begin{array}{l}0.00 \pm 0.72^{\mathrm{b}} \\
(\mathrm{n}=2,234)\end{array}$ & $\begin{array}{c}0.01 \pm 1.04^{\mathrm{b}} \\
(\mathrm{n}=224)\end{array}$ & $\begin{array}{c}0.90 \pm 0.96^{\mathrm{a}} \\
(\mathrm{n}=224)\end{array}$ \\
\hline Young bulls from B & $44 \%$ & $\begin{array}{l}0.00 \pm 0.71^{\mathrm{b}} \\
(\mathrm{n}=1.282)\end{array}$ & $\begin{array}{c}0.02 \pm 1.06^{\mathrm{b}} \\
(\mathrm{n}=131)\end{array}$ & $\begin{array}{c}0.99 \pm 0.99^{\mathrm{a}} \\
(\mathrm{n}=131)\end{array}$ \\
\hline Young bulls from $\mathrm{C}$ & $50 \%$ & $\begin{array}{l}0.00 \pm 0.74^{\mathrm{b}} \\
(\mathrm{n}=3,602)\end{array}$ & $\begin{array}{c}0.07 \pm 1.12^{\mathrm{b}} \\
(\mathrm{n}=362)\end{array}$ & $\begin{array}{c}1.12 \pm 1.02^{\mathrm{a}} \\
(\mathrm{n}=362)\end{array}$ \\
\hline
\end{tabular}

a,b Means followed by different superscript letters differed $(P<0.001)$.

${ }^{1}$ No selection $=\mathrm{CTL}$; random selection $=$ RD SELU; genomic selection $=$ MS SELU.

${ }^{2} \mathrm{GEBV}=$ genomically enhanced breeding value

${ }^{3}$ Random preselection among young bulls (10\% retained).

${ }^{4}$ Genomic preselection among young bulls (10\% retained).

deviation in the MS SELU scenario with the SD in the random case (RD SELU) and notice that it was actually reduced after mimicking a GPS. Therefore, we checked that, in our simulations, the MS distribution clearly deviated from the expected one, violating the mixed linear model hypothesis for unbiased and more accurate estimations of breeding values.

\section{Effect of Using a Subset of Data After Genomic Selection on the International Evaluations}

Scenarios MS SELU-A, -B, or -C and scenarios RD SELU-A, -B, or -C provided estimates of bias in I-EBV after preselection of data based on MS or random terms. Based on adjusted bias estimates, Table 4 compares the direction and magnitude of bias across cohorts (YB, their sires, and half-sibs), traits (A, B, C) and country strategies (GPS in A, B, or C). Table 5 focuses on the GPS consequences among the YB retained from genomic selection based on bias assessments in genetic SD unit. The YB from the other countries might also be affected, but differently according to the trait: (1) affected by GPS (Figure 1), and (2) nonaffected by GPS (Figure 2). For example, the MS SELU-A scenario was considered when preselected but unbiased N-EBV for trait A were sent to the Interbull Centre. Figures 1 and 2 present boxplots of the bias in I-EBV, expressed in genetic $\mathrm{SD}$ of the evaluated trait, among $\mathrm{YB}$ from country A, B, or C for trait A or B.

The I-EBV of YB from the country with GPS (A in our illustration) were systematically underestimated on all traits. They were especially underestimated on traits evaluated in countries without GPS (Table 4). In fact, bias estimates ranged from about one-tenth to one-quarter of the genetic SD of the trait (Table 5). Note that these values were significantly different from bias estimates in RD SELU scenarios, showing that a preselection based on nonrandom criteria, which are MS terms responsible for systematic errors independent from the decrease of information in MACE. Moreover, I-EBV of YB from countries without GPS (B and C in our illustration) tended to be slightly overestimated (Table 4). In fact, YB retained from genomic selection were penalized for all traits.

To assess the importance of these bias for selection decisions, changes in the top 100 ranked bulls were observed. Compared with the CTL scenario, rerankings occurred. Correlations (Pearson) between top 100 rankings in CTL and MS SELU scenarios dropped to 0.80. Among the top $100 \mathrm{YB}$ and older bulls, the proportion of YB from the country with GPS decreased (Table 6) for all traits, affected or not by GPS. On average, 25\% of the YB may disappear from the top list. They were replaced by YB from the other countries. Their proportion in the top list increased from 13 to $30 \%$ according to the considered trait.

Table 4 also indicates that not only the YB retained after a genomic selection step and their contemporaries were affected, but also their sires and half-sibs. Their breeding values tended to be underestimated for traits evaluated in countries without GPS (off-diagonal elements in Table 4) and close to or slightly larger than zero for the trait evaluated in the country with GPS (diagonal elements in Table 4). Generally, bias estimates among the cohorts of sires and half-sibs of YB were statistically different from zero. Sires of YB seemed to be more affected than half-sibs of YB. However, the magnitude of bias was very small compared with the magnitude of bias among YB. These simulations showed that bias was created in I-EBV for all traits among the YB retained from genomic selection and their contemporaries and could be communicated to their relatives to a lesser extent, leading to obviously unfair international comparisons. 
Table 4. Adjusted bias in I-EBV ${ }^{1}$ for trait A, B, or C and among different cohorts of animals in the population to evaluate under MS SELU A, B, C and MS SELB ${ }^{3}$ A, B, C scenarios

\begin{tabular}{|c|c|c|c|c|c|c|c|c|}
\hline \multirow{2}{*}{ Cohort } & \multirow{2}{*}{$\begin{array}{l}\text { Trait } \\
\text { affected } \\
\text { by GPS }\end{array}$} & \multirow{2}{*}{$\begin{array}{l}\text { Size of the } \\
\text { cohort }(\times 10 \\
\text { repetitions })\end{array}$} & \multicolumn{3}{|c|}{ Scenarios MS SELU } & \multicolumn{3}{|c|}{ Scenarios MS SELB } \\
\hline & & & \multicolumn{3}{|c|}{ Evaluated trait } & \multicolumn{3}{|c|}{ Evaluated trait } \\
\hline \multirow[t]{3}{*}{ YB from $\mathrm{A}$} & $\mathrm{A}$ & 224 & $-0.22^{5}$ & -0.52 & -0.46 & -4.88 & -2.08 & -3.49 \\
\hline & $\mathrm{B}$ & 2,234 & $0.08^{6}$ & 0.14 & 0.08 & 0.07 & 0.15 & 0.10 \\
\hline & $\mathrm{C}$ & 2,234 & 0.15 & 0.15 & 0.29 & 0.11 & 0.11 & 0.19 \\
\hline & $\mathrm{C}$ & 1,282 & -0.03 & 0.35 & 0.14 & -0.04 & 0.26 & 0.11 \\
\hline \multirow[t]{3}{*}{ YB from $\mathrm{C}$} & $\mathrm{A}$ & 3,602 & 0.07 & 0.03 & 0.10 & 0.07 & 0.03 & 0.08 \\
\hline & B & 3,602 & -0.01 & 0.02 & 0.03 & -0.02 & 0.02 & 0.03 \\
\hline & $\mathrm{C}$ & 362 & -0.46 & -0.46 & -0.75 & -0.87 & -0.96 & -4.53 \\
\hline \multirow[t]{3}{*}{ Sires of YB } & $\mathrm{A}$ & 99 & -0.03 & -0.09 & -0.17 & -0.04 & -0.08 & -0.12 \\
\hline & $\mathrm{B}$ & & -0.13 & -0.02 & -0.13 & -0.11 & -0.02 & -0.09 \\
\hline & $\mathrm{C}$ & & -0.28 & -0.27 & 0.40 & -0.20 & -0.18 & 0.24 \\
\hline
\end{tabular}

${ }^{1} \mathrm{I}-\mathrm{EBV}=$ international EBV as multiple-trait across country evaluation solutions.

${ }^{2}$ Mendelian sampling with preselected and unbiased national EBV (when country A, B, or C sent preselected data to Interbull Centre after GPS).

${ }^{3}$ Mendelian sampling with preselected and biased national EBV (when A, B, or C country sent to Interbull Centre preselected and biased data after GPS).

${ }^{4}$ Genomic preselection.

${ }^{5}$ Numbers in bold represent when bias was estimated for the trait evaluated in the country implementing GPS (not in bold for the traits evaluated in other countries without GPS).

${ }^{6}$ Numbers in italic corresponds to the cohort of young bulls (YB) from countries without GPS (contemporaries of young bulls retained by GPS).

\section{Effect of Using Biased Breeding Values Due to Genomic Selection in the International Evaluations}

The results from the MS SELB-A, -B, and -C scenarios were analyzed to highlight the effect of using preselected and biased breeding values into the inter- national evaluations. For illustration, Figures 3 and 4 present the distribution of bias in I-EBV (expressed in genetic SD unit) among YB when MS SELB-A was simulated. In that case, country A sent biased N-EBV to the Interbull Centre for only YB retained from genomic selection. In Figure 3, boxplots of bias are dis-

Table 5. Bias estimates in I-EBV ${ }^{1}$ expressed in standard deviation unit for trait A, B, or C among young bulls (YB) from the country with genomic preselection (GPS) MS SELU² and MS SELB ${ }^{3}$ scenarios

\begin{tabular}{|c|c|c|c|c|c|c|c|}
\hline \multirow[b]{3}{*}{ Cohort } & \multirow{3}{*}{$\begin{array}{l}\text { Trait } \\
\text { affected } \\
\text { by GPS }\end{array}$} & \multicolumn{3}{|c|}{ Scenarios MS SELU } & \multicolumn{3}{|c|}{ Scenarios MS SELB } \\
\hline & & \multicolumn{3}{|c|}{ Evaluated trait } & \multicolumn{3}{|c|}{ Evaluated trait } \\
\hline & & $\mathrm{A}$ & B & $\mathrm{C}$ & A & B & $\mathrm{C}$ \\
\hline YB from $A$ & $\mathrm{~A}$ & $-0.01^{4}$ & -0.07 & -0.07 & 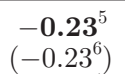 & $\begin{array}{c}-0.25^{4} \\
\left(-0.17^{5}\right)\end{array}$ & $\begin{array}{c}-0.25^{4} \\
\left(-0.18^{4}\right)\end{array}$ \\
\hline YB from B & $\mathrm{B}$ & -0.13 & -0.02 & -0.09 & $\begin{array}{c}-0.29^{4} \\
\left(-0.16^{5}\right)\end{array}$ & $\begin{array}{c}-\mathbf{0 . 2 4} 4^{4} \\
\left(-0.22^{5}\right)\end{array}$ & $\begin{array}{r}-0.27^{4} \\
\left(-0.18^{5}\right)\end{array}$ \\
\hline
\end{tabular}

${ }^{1} \mathrm{I}$-EBV = international EBV as multiple-trait across country evaluation solutions.

${ }^{2}$ Mendelian sampling with preselected and unbiased national EBV (when country A, B, or C sent to Interbull Centre preselected data after GPS).

${ }^{3}$ Mendelian sampling with preselected and biased national EBV (when country A, B, or C sent to Interbull Centre preselected and biased data after GPS).

${ }^{4}$ Numbers in bold corresponds to the cohort of young bulls retained by GPS when bias was estimated for the trait evaluated in their country of origin (not in bold for the traits evaluated in other countries without genomic preselection).

${ }^{5}$ Observed bias due to both effects: sending preselected and biased data.

${ }^{6}$ Assumed part of the bias only due to the effect of sending biased data. 


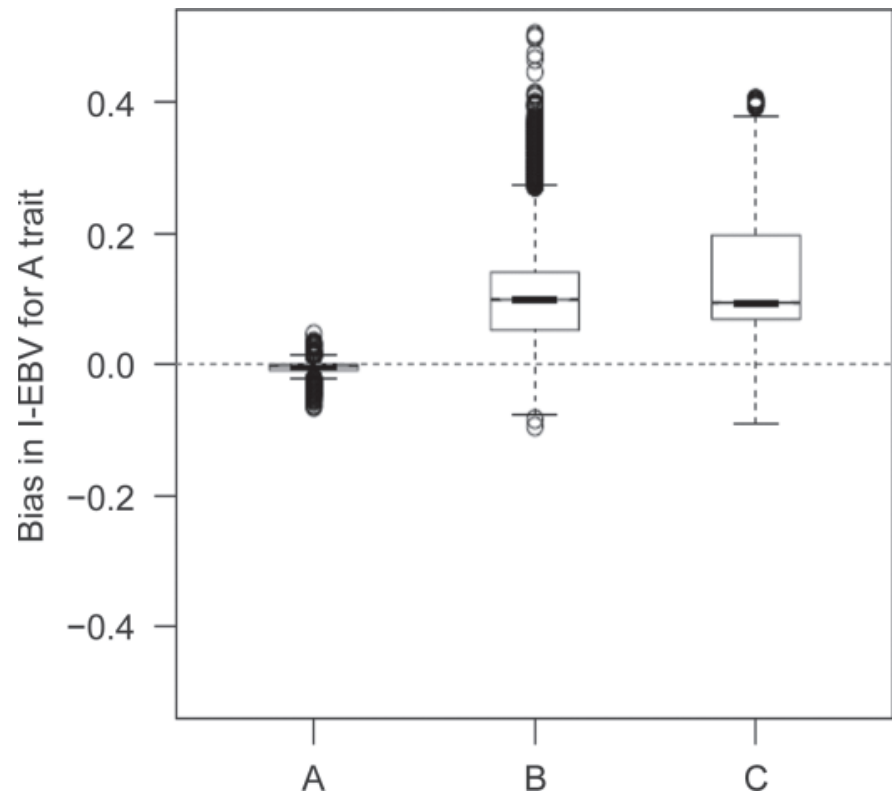

Figure 1. Bias distribution in international EBV (I-EBV) (expressed in genetic standard deviation of the trait) among young bulls from countries A, B, and C for trait A when evaluated with preselected data (after genomic preselection) for young bulls (the Mendelian sampling with preselected and unbiased national EBV scenario A, MS SELU-A).

played for trait A (i.e., for the trait with preselected and biased data after a GPS). In Figure 4, boxplots of bias are displayed for trait $\mathrm{B}$, which was evaluated based on a complete set of performances, among preselected (from country A) and not preselected YB (from country B and C). Compared with results under MS SELU scenarios, estimates of bias tended to be pushed down according to the negative bias transmitted from the national level.

Table 5 not only provides the bias estimates in MS SELU and MS SELB scenarios, but also the effect of sending biased data $\left(\hat{E}_{3}=\hat{b}^{M S S E L B}-\hat{b}^{M S} S E L U\right.$, in parenthesis) besides preselected data. Bias was clearly

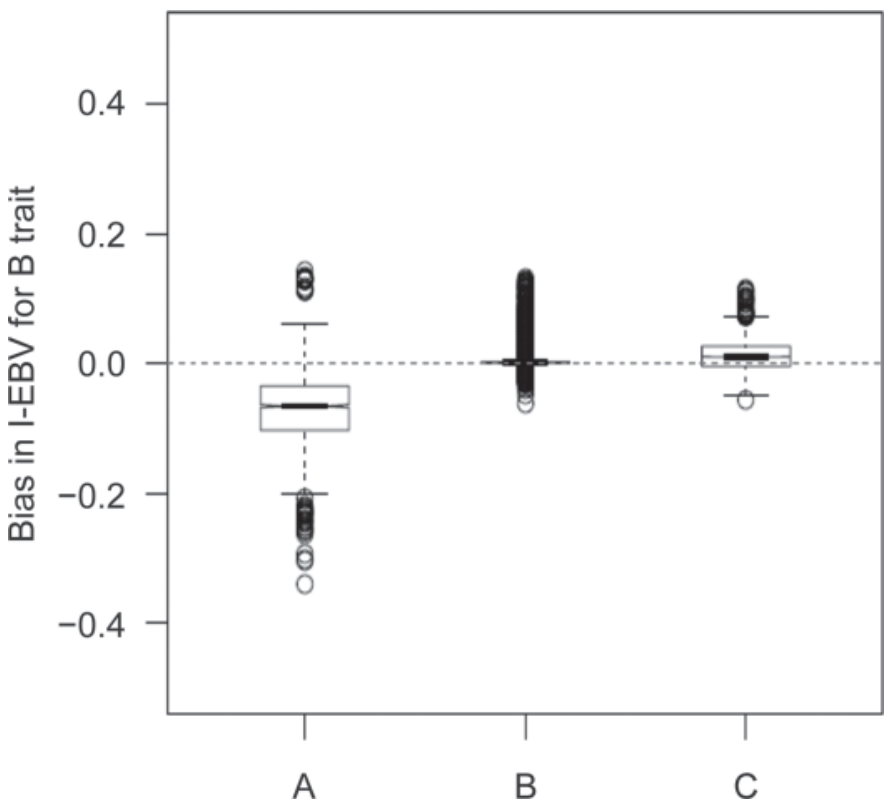

Figure 2. Bias distribution in international EBV (I-EBV) (expressed in genetic standard deviation of the trait) among young bulls from countries A, B, and C for trait B (evaluated in country without genomic preselection) in the Mendelian sampling with preselected and unbiased national EBV scenario A (MS SELU-A).

transmitted from the national to the international level. Among YB retained by genomic selection (from country A in Figure 3), the average bias at the national level was the same as the bias estimated at the international level for the trait evaluated in the country doing GPS (trait A in our illustration).

Bias was also transmitted from one trait to another correlated trait, but to a lesser extent. The part of bias due to the use of biased $\left(\hat{E}_{3}\right)$ data was always smaller for the correlated trait. The magnitude of bias seemed to be the function of the genetic correlations between traits; the higher the correlation is, the larger the bias transmitted.

Table 6. Decrease in the proportion of young bulls (YB) from the country with genomic preselection (GPS) among the 100 top sires for trait A, B, or C

\begin{tabular}{llccc}
\hline Cohort & Scenario & Trait A & Trait B & Trait C \\
\hline YB from A & MS SELU $^{1} \mathrm{~A}$ & $-1 / 13^{2}$ & $-1 / 2$ & $-1 / 7$ \\
& MS SELB A & $-8 / 13$ & $-2 / 2$ & $-5 / 7$ \\
YB from B & MS SELU B & $-1 / 6$ & $-3 / 15$ & $-2 / 8$ \\
& MS SELB B & $-3 / 6$ & $-8 / 15$ & $-7 / 8$ \\
YB from C & MS SELU C & $-8 / 19$ & $-7 / 21$ & $-5 / 34$ \\
& MS SELB C & $-10 / 19$ & $-10 / 20$ & $-11 / 34$ \\
\hline
\end{tabular}

${ }^{1}$ Mendelian sampling with preselected and unbiased national EBV (when country A, B, or C sent to Interbull Centre preselected data after a GPS).

${ }^{2}$ Denominator represents the total number of YB from the country with GPS included in the 100 top sires under the control scenario.

${ }^{3}$ Mendelian sampling with preselected and biased national EBV (when country A, B, or C sent to Interbull Centre preselected and biased data after a GPS). 


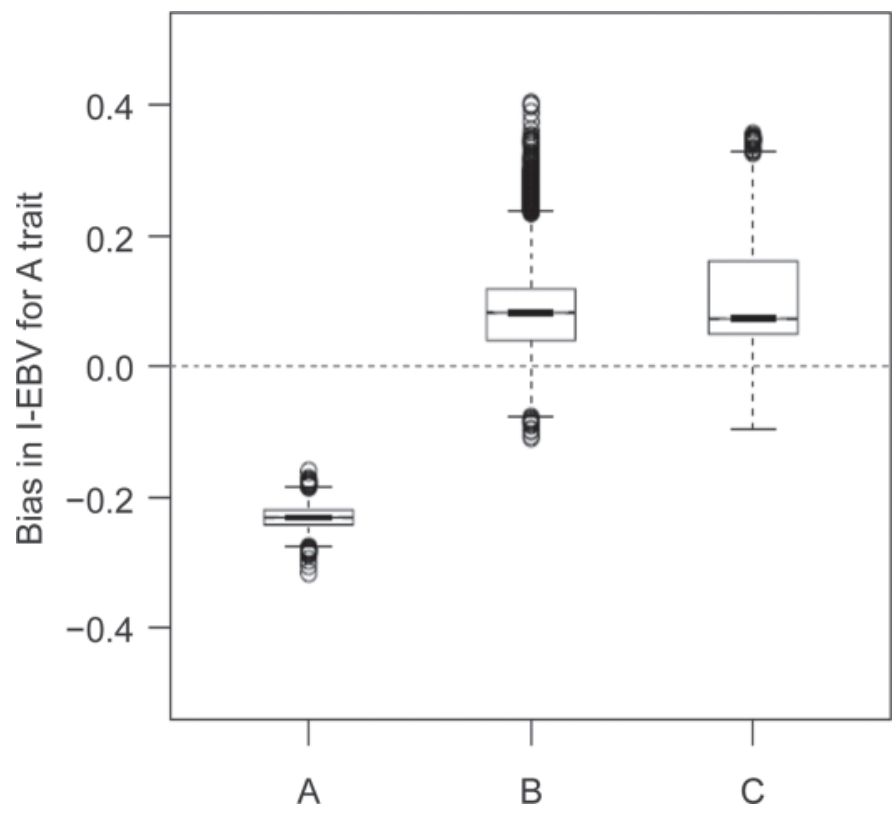

Figure 3. Bias distribution in international EBV (I-EBV) (expressed in genetic standard deviation of the trait) among young bulls from countries $\mathrm{A}, \mathrm{B}$, and $\mathrm{C}$ for trait $\mathrm{A}$ when evaluated with preselected (after genomic preselection) and biased data for young bulls (the Mendelian sampling with preselected and biased national EBV scenario A, MS SELB-A).

Again, YB retained by genomic selection were the most penalized. They were more severely underestimated in MS SELB scenarios than in MS SELU scenarios for all traits (Table 4). Bias due to the use of biased data seemed more harmful than bias due to preselected data. It follows that the proportion of $\mathrm{YB}$ from the country with GPS clearly decreased among the top 100 bulls in favor of YB from other countries (Table 6). However, bias estimates among relatives of YB are of the same extent as in MS SELU scenarios. The transmission of $\hat{E}_{3}$ was probably buffered due to the high reliability of I-EBV among these cohorts.

Finally, YB from country A and YB from country $\mathrm{B}$ were especially affected when country $\mathrm{C}$ sent preselected and potentially biased data after the genomic selection step. Generally, the proportion in the top 100 ranked bulls was the largest for bulls from country C, whatever the trait. Bulls from country $\mathrm{C}$ have been used more in other countries and, therefore, these YB may have more relatives in the various countries, which could explain the difference of bias magnitude across countries independent from genetic parameters and size of the cohort of interest.

\section{DISCUSSION}

The comparison of I-EBV resulting from the various simulated scenarios shows that sending preselected and

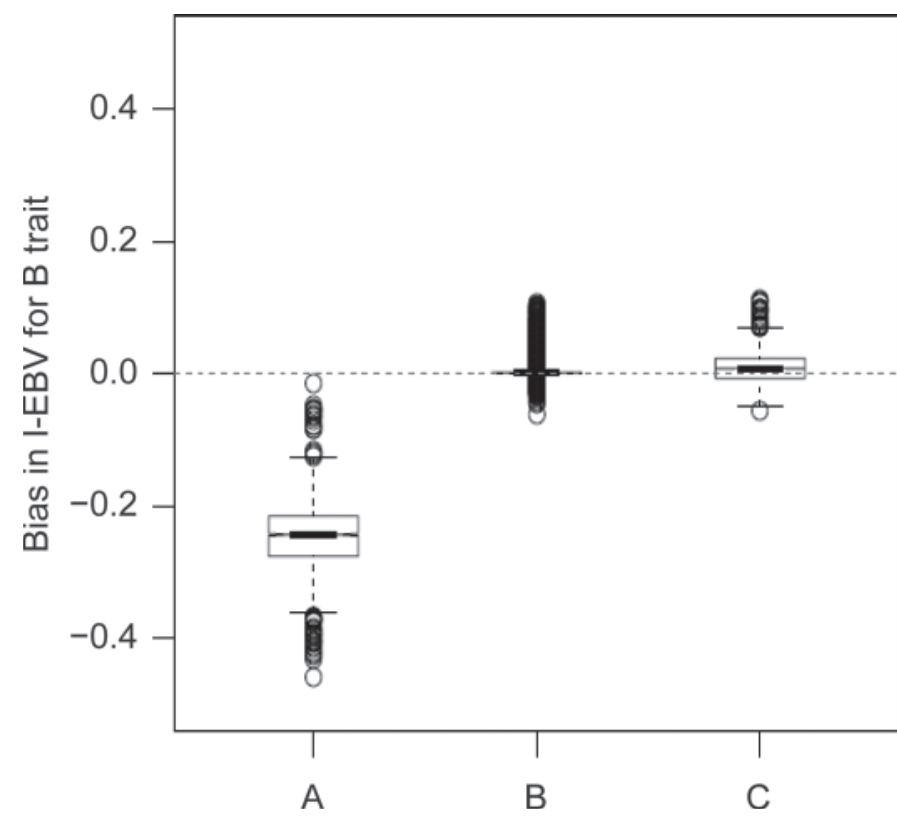

Figure 4. Bias distribution in international EBV (I-EBV) (expressed in genetic standard deviation of the trait) among young bulls from countries A, B, and C for trait B (evaluated in country without genomic preselection) in Mendelian sampling with preselected and biased national EBV scenario A (MS SELB-A).

potentially biased data to the Interbull Centre created biased international evaluations. Sending preselected but unbiased N-EBV was shown to be much less harmful than sending preselected and biased data. The MACE solutions were not only affected for YB after a GPS, but also for their contemporaries from countries which were not implementing GPS. Such selected YB were actually penalized for all traits (i.e., for the trait evaluated in their country of origin as well as for traits evaluated in other countries). Such bias tended to be transmitted to relatives in the population; this obviously led to unfair international comparisons of the country implementing GPS.

Mimicking decisions based on genomic information and their effects on national breeding values required approximations in the simulation of the MS terms and of the bias term in N-EBV for YB. Thus, bias assessments were not an accurate measure of the error magnitude which could be observed in real conditions. For example, when mimicking the GPS, genomic reliabilities were underestimated, which could buffer bias magnitude. Actually, many traits in many countries have GEBV reliabilities higher than $50 \%$. In the future, this level is expected to be even higher with larger joint reference population, which should strengthen the simulated trends. At the same time, interactions between only 3 countries were considered. A balance had to be found in representing a compromise between 
a realistic and a simple scenario to at least highlight and understand the main trends. The effects of genomic preselection on the international evaluations were systematically observed in various and repeated scenarios. The simulation of various GPS strategies on 3 different traits among 3 cohorts of $\mathrm{YB}$, and the fact that I-EBV remained unbiased after a random preselection of data, actually showed that the measured bias from simulated data was not due to a random error.

Simplified designs of simulation facilitated the understanding of the mechanisms behind the bias. Genetic preselection leads first to the absence of observations for the culled animals so that the distribution of the MS terms for phenotyped animals differs from the expected one, with an average different from 0 . This violates the BLUP, and therefore the MACE hypotheses. At the national level, Patry and Ducrocq (2011b) already showed that genetic evaluations for the bulls retained by GPS were underestimated, and therefore disadvantaged compared with other contemporary groups. The same trend was observed at the international level; the preselection of data in one country based on genomic information also penalized the YB retained by GPS. In fact, they were not only underestimated, I-EBV of their contemporaries were also overestimated. Second, due to inconsistencies between the distributions of N-EBV among YB from various countries, biases were created among the contemporary cohort of $\mathrm{YB}$, retained by GPS or not, and whatever the trait considered as a reference during the genetic evaluation. Third, when biased N-EBV were used as performances in MACE, bias was clearly transmitted from the national level to the international level. Such an underestimation was then transmitted from one trait to another through the matrix of genetic variance-covariances. Finally, any systematic error observed among the YB, retained by GPS and their contemporaries, was communicated to their relatives. The magnitude of bias might be buffered by the coefficients of genetic relationships. These animals also have performances in various countries and their I-EBV have a high reliability, which makes them less sensitive to any bias.

It is feared that the direction and magnitude of the bias could be difficult to predict when various GPS strategies are implemented across countries at the same time. All countries participating in international evaluations could be affected, whatever the GPS strategy, but in an uncontrolled way. Disorganization is certain, and it is essential to prevent it from happening.

First, GPS must be accounted for in national evaluation models. All culled and selected candidates should be included in national evaluations according to various procedures, for example, single-step (Aguilar et al., 2010; Christensen and Lund, 2010; Legarra and Ducrocq, 2012) or multi-step procedures (Ducrocq and Liu, 2009; Mäntysaari and Strandén, 2010; Stoop et al., 2011). Adaptations of these approaches are still required to be implemented routinely. It is possible to get breeding values for all candidates, including the culled ones, which could then be sent to the Interbull Centre.

It follows that all the BLUP solutions, adjusted for GPS, would include genomic information besides the pedigree-type and phenotypic information. Such additional information could create redundancy in the MACE analyses. Moreover, I-EBV are required for inclusion of foreign bulls in the reference population to derive future genomic predictions. In that case, using I-EBV enhanced with genomic information as observed performances to estimate genomic effects might generate dependency between observed and predicted data and affect the quality of future genomic evaluations. Moreover, the number of candidates (and, thus, N$\mathrm{EBV}$ ) to include in MACE may dramatically increase with the spread of genomic tools worldwide. Managing such a huge quantity of data may also become a problem at the Interbull level. Various options could be proposed to deal with the dependency between classical and genomic evaluations:

- Option 1: Data free from genomic information (e.g., daughter yield deviation; DYD; VanRaden, 2012) could be to send to Interbull Centre. By definition, DYD are weighted averages of daughter records corrected for all fixed effects and breeding values of their dam (VanRaden and Wiggans, 1991). They can be derived from the national genetic evaluations as a coproduct. Values for DYD were widely used in QTL mapping (Georges et al., 1995), in validating national genetic trends (Boichard et al., 1995), and in proposing improvements to international bull comparisons (Ducrocq et al., 2003). They can now be derived from single-step or multi-step procedures of national evaluations which consider genomic information. Here, the advantage of DYD would be to isolate the phenotypic information from not only the pedigreetype but also the genomic ones. However, as often discussed among countries participating in international evaluations, this would be more difficult for countries using test-day models to provide an appropriate weight (Liu et al., 2004). It would also be complicated for the Interbull Centre to check the homogeneity of DYD across countries.

- Option 2: A single-step evaluation, including data from all genotyped candidates and nongenotyped animals, could be run at the international level 
to properly account for all sources of information. This solution is far from being realistic in the short term, as single-step procedures are still under development at the national level and face some computing challenges, especially for very large cohorts of genotyped animals.

- Option 3: Genetic group effect, as applied for base population (Quaas, 1988) but for contemporary animals undergoing GPS, could be defined and estimated, which could also alleviate the international genetic evaluations from the constraints of size of data set.

Adapting the Interbull validation tests (Boichard et al., 1995; Fikse et al., 2003) and checking the quality of the data sent by the participating countries might also be required to control the completeness and unbiasedness of N-EBV regarding GPS, possibly based on the distribution of MS estimates as implemented.

\section{CONCLUSIONS}

This study assessed the bias at the international level due to GPS. It was shown that using selected and possibly biased N-EBV affected the quality of international genetic evaluations delivered by the Interbull Centre. This, obviously, led to unfair international comparisons. The bulls which underwent GPS were the most penalized ones. Moreover, biases were created among their contemporaries from other countries and propagated to their relatives, as well as to the correlated traits, affecting international rankings on all traits. The consequences on international genetic evaluations would be difficult to predict in complex and heterogeneous situations considering the diversity of breeding practices and policies within and between countries. Accounting for GPS at the national level and providing all available information to the Interbull Centre are of high relevance regarding the impacts on the classical international rankings or on the quality of the future genomic predictions based on shared reference populations. The method for international evaluation on the type of data sent to the Interbull Centre should also be adapted to consider and make beneficial the newly available genomic information.

\section{ACKNOWLEDGMENTS}

We gratefully thank the Interbull Centre (Uppsala, Sweden) for allowing the use of N-EBV and for making available tools to implement the MACE methodology for this study. We also thank the Department of Animal Breeding and Genetics at the Swedish University of Agricultural Sciences (Uppsala, Sweden), the Production Trait European Joint Evaluation [Associazione Nazionale Allevatori Bovini Razza Frisona Italiana (ANAFI), Cremona, Italy; Coöperatie Rundvee Verbetering (CRV), Arnhem, the Netherlands; Nordisk Avlsværdivurdering (NAV), Denmark; University of Liège, Belgium; Vereinigte Informationssysteme Tierhaltung (VIT), Verden, Germany], and the Methodological Approaches and Implementation of Genomic Selection (AMASGEN) working groups for their useful remarks. Financing of the AMASGEN project (Jouy-en-Josas, France) by the Agence Nationale de la Recherche and the Apis Gène Society (APIS-GENE, Paris, France) is gratefully acknowledged. We also thank the reviewers for their comments and suggestions.

\section{REFERENCES}

Aguilar, I., I. Misztal, D. L. Johnson, A. Legarra, S. Tsuruta, and T. J. Lawlor. 2010. A unified approach to utilize phenotypic, full pedigree, and genomic information for genetic evaluation of Holstein final score. J. Dairy Sci. 93:743-752.

Boichard, D., B. Bonaiti, A. Barbat, and S. Mattalia. 1995. Three methods to validate the estimation of genetic trend for dairy cattle. J. Dairy Sci. 78:431-437.

Christensen, O. F., and M. Lund. 2010. Genomic prediction when some animals are not genotyped. Genet. Sel. Evol. 42:2.

Ducrocq, V., I. Delaunay, D. Boichard, and S. Mattalia. 2003. A general approach for international genetic evaluations robust to inconsistencies of genetic trends in national evaluations. Interbull Bull. 30:101-111.

Ducrocq, V., and Z. Liu. 2009. Combining genomic and classical information in national BLUP evaluations. Interbull Bull. 40:172-177.

Fikse, W. F., L. Klei, Z. Liu, and P. G. Sullivan. 2003. Procedure for validation of trends in genetic variances. Interbull Bull. 31:30-36.

Georges, M., D. Nielsen, M. Mackinnon, A. Mishra, R. Okimoto, A. T. Pasquino, L. S. Sargeant, A. Sorensen, M. R. Steele, X. Zhao, J. E. Womack, and I. Hoeschele. 1995. Mapping quantitative trait loci controlling milk production in dairy cattle by exploiting progeny testing. Genetics 139:907-920.

Henderson, C. R. 1975. Best Linear Unbiased Estimation and Prediction under a selection model. Biometrics 31:423-427.

Legarra, A., I. Aguilar, and I. Misztal. 2009. A relationship matrix including full pedigree and genomic information. J. Dairy Sci. 92:4656-4663.

Legarra, A., and V. Ducrocq. 2012. Computational strategies for national integration of phenotypic, genomic, and pedigree data in a single-step best linear unbiased prediction. J. Dairy Sci. 95:46294645 .

Liu, Z., F. Reinhardt, A. Bünger, and R. Reents. 2004. Derivation and calculation of approximate reliabilities and daughter yield-deviations of a random regression test-day model for genetic evaluation of dairy cattle. J. Dairy Sci. 87:1896-1907.

Loberg, A., H. Jorjani, and J. W. Dürr. 2011. Validation of genomic national evaluations. Interbull Bull. 44:62-66.

Mäntysaari, E. A., and I. Strandén. 2010. Use of bivariate EBV-DGV model to combine genomic and conventional breeding value estimations. In Proc. 9th WCGALP, Leipzig, Germany. German Society for Animal Science, Giessen, Germany.

Misztal, I., A. Legarra, and I. Aguilar. 2009. Computing procedures for genetic evaluation including phenotypic, full pedigree, and genomic information. J. Dairy Sci. 92:4648-4655.

Patry, C., and V. Ducrocq. 2011a. Accounting for genomic pre-selection in national BLUP evaluations in dairy cattle. Genet. Sel. Evol. 43:30 
Patry, C., and V. Ducrocq. 2011b. Evidence of biases in genetic evaluations due to genomic preselection in dairy cattle. J. Dairy Sci. 94:1011-1020.

Quaas, R. L. 1988. Additive genetic model with groups and relationships. J. Dairy Sci. 71:1338-1345.

Schaeffer, L. R. 1994. Multiple-country comparison of dairy sires. J. Dairy Sci. 77:2671-2678.

Stoop, W. M., H. Eding, M. L. van Pelt, and G. de Jong. 2011. Combining genomic and conventional data in the Dutch national evaluation. Interbull Bull. 44:169-172.
VanRaden, P. M., and G. R. Wiggans. 1991. Derivation, calculation, and use of national animal model information. J. Dairy Sci. $74: 2737-2746$

VanRaden, P. 2012. Avoiding bias from genomic pre-selection in converting daughter information across countries. Interbull Bull. 45:1-5. 